\title{
Desenvolvimento de Gramados Submetidos à Aplicação de Retardadores de Crescimento em Diferentes Condições de LUMINOSIDADE ${ }^{1}$
}

\author{
Development of Turfgrass Submitted to Application of Growth Regulators under Different Light \\ Conditions
}

\author{
MACIEL, C.D.G. ${ }^{2}$, POLETINE, J.P. ${ }^{3}$, RAIMONDI, M.A. ${ }^{3}$, RODRIGUES, M.. ${ }^{4}$ RIBEIRO, R.B. ${ }^{5}$, \\ COSTA, R.S. ${ }^{5}$ e MAIO, R.M.D. ${ }^{5}$
}

\begin{abstract}
RESUMO - Com o objetivo de avaliar o desenvolvimento das gramas Bermuda (Cynodon dactylon), Esmeralda (Zoysia japonica) e São Carlos (Axonopus compressus) submetidas a subdoses de herbicidas e reguladores de crescimento em duas condições de luminosidade, três experimentos foram conduzidos em estufa plástica em Paraguaçu Paulista-SP, de setembro a dezembro de 2006. Para cada espécie de grama, o delineamento experimental utilizado foi o inteiramente casualizado, em esquema fatorial $6 \times 2$, com quatro repetições; foram estudados dois reguladores de crescimento (trinexapac-ethyl e ethephon) e três herbicidas aplicados em subdoses (clethodim, imazethapyr e metsulfuron-methyl) e uma testemunha sem aplicação. Todos os tratamentos foram avaliados em duas condições de luminosidade, representadas por ausência de sombreamento $(0 \%)$ e sombreamento parcial (50\%). Os resultados obtidos aos 63 dias após aplicação evidenciaram que o trinexapacethyl (250 $\left.\mathrm{g} \mathrm{ha}^{-1}\right)$, clethodim $\left(12 \mathrm{~g} \mathrm{ha}^{-1}\right)$ e imazethapyr (12 $\left.\mathrm{g} \mathrm{ha}^{-1}\right)$ apresentaram-se como os melhores retardadores do desenvolvimento vegetativo das gramas C. dactylon (65,8, 29,6 e $18,7 \%), Z$. japonica (66,2, 40,7 e 49,7\%) e A. compressus $(56,5,10,3$ e 17,2\%) e da emissão de inflorescências de C. dactylon (100,0, 95,9 e 89,6\%), em baixa e alta luminosidade. Os tratamentos ethephon (300 $\left.\mathrm{g} \mathrm{ha}^{-1}\right)$ e metsulfuron-methyl (3,6 $\mathrm{g}^{\text {ha-1) }}$ destacaram-se como as melhores opções para redução do desenvolvimento vegetativo (15,5 e 26,7\%) e da matéria seca das raízes (34,7 e 33,9\%) da grama São Carlos (A. compressus), quando comparados ao trinexapac-ethyl. Para gramados de C. dactylon e $Z$. japonica, em condições em que a preservação da estética é fundamental, os herbicidas clethodim e imazethapyr podem substituir o regulador de crescimento trinexapac-ethyl, em razão do menor dano visual no gramado. Novos estudos com reguladores de crescimentos e subdoses de herbicidas devem ser conduzidos com outras gramas cultivadas, de forma a viabilizar recomendações eficientes e seguras, com embasamento em informações científicas geradas em condições brasileiras.
\end{abstract}

Palavras-chave: regulador vegetal, herbicida, Cynodon dactylon, Zoysia japonica, Axonopus compressus.

\begin{abstract}
In order to evaluate the development of the grass species Bermuda (Cynodon dactylon), Emerald (Zoysia japonica) and Broadleaf Carpetgrass (Axonopus compressus) submitted to herbicide sub doses and growth regulators undertwo light conditions, three experiments were conducted in a greenhouse at Paraguaçu Paulista from September to December, 2006. For each grass species, the experimental design used was completely randomized, in a factorial scheme $6 \times 2$, with four replications, using two growth regulators (trinexapac-ethyl and ethephon), three
\end{abstract}

1 Recebido para publicação em 18.7.2010 e na forma revisada em 6.5.2011.

2 Engo-Agr ${ }^{0}$., D.Sc., Professor Adjunto do Dep. de Ciências Agronômicas, Universidade Estadual de Maringá - UEM, Campus

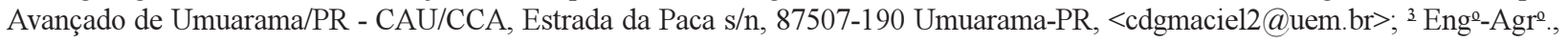
Aluno do Programa de Pós-Graduação em Agronomia na área de Proteção de Plantas, Universidade Estadual de Maringá - NAPD/ UEM, Av. Colombo, 5790, 87020-9000 Maringá-PR; ${ }^{4}$ Acadêmicos do Curso de Agronomia, Escola Superior de Agronomia de Paraguaçu Paulista - FUNGE/ESAPP, Rua Prefeito Jayme Monteiro, 791, 19700-000 Paraguaçu Paulista-SP, ${ }^{5}$ Engo-Agr ${ }^{0}$., Empresa Gramar - Gramados Marília Ltda, Alameda Santa Carolina, 420, 17514-830 Marília-SP.

Planta Daninha, Viçosa-MG, v. 29, n. 2, p. 383-395, 2011 
herbicides applied in sub doses (clethodim, imazethapyr and metsulfuron-methyl), and a check without application. All treatments were evaluated undertwo light conditions, represented by plastic greenhouse (total light) and plastic greenhouse + shading 50\% (partial light). The results obtained at 63 days after application showed that trinexapac-ethyl (250 $\left.\mathrm{g} \mathrm{ha}^{-1}\right)$, clethodim (12 $\left.\mathrm{g} \mathrm{ha} \mathrm{h}^{-1}\right)$ and imazethapyr $\left(12 \mathrm{~g} \mathrm{ha}^{-1}\right)$ were the best growth regulators for the vegetative development of $\mathbf{C}$. dactylon (65.8, 29.6 and 18.7\%), Z. japonica (66.2, 40.7 and 49.7\%) and A. compressus (56.5, 10.3 and $17.2 \%)$ grasses and for inflorescence emission of C. dactylon (100.0, 95.9 and 89.6\%), under high

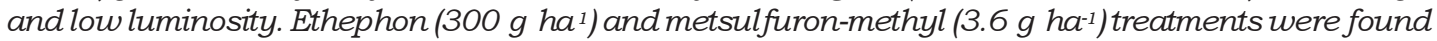
to be the best options for reducing vegetative development (15.5 and 26.7\%) and root dry matter (34.7 and 33.9\%) of São Carlos grass (A. compressus), compared to trinexapac-ethyl. For C. dactylon and $Z$. japonica turf grass, under conditions where aesthetics preservation is fundamental, clethodim and imazethapyrmay substitute trinexapac-ethyl growth regulator on account of the smaller visual damage in the grass. Further studies with growth regulators and herbicide sub doses must be conducted with other cultivated grasses to enable efficient and safe recommendations, based on scientific information generated from Brazilian conditions.

Keywords: plant regulator, herbicide, Cynodon dactylon, Zoysia japonica, Axonopus compressus.

\section{INTRODUÇÃO}

Uma verdadeira revolução técnica vem acontecendo com os gramados dos principais estádios e clubes de futebol, golfe e tênis em todo o mundo, principalmente devido ao alto profissionalismo que envolve o esporte. No Brasil, o interesse em modernas técnicas de manutenção aumentou, não sendo difícil encontrar ambientes com gramados de excelente qualidade. Com isso, termos técnicos como "corte helicoidal", "aeração e descompactação", "overseeding", "cobertura top dresser" já fazem parte do dia a dia de empresas de jardinagem, que contam com profissionais especializados para o trabalho no segmento de gramados.

Entretanto, os gramados podem ser utilizados em diversos locais com os diferentes propósitos; nas mais diversas situações, o principal fator do custo de manutenção é o corte na altura adequada (Freitas et al., 2002; Maciel et al., 2006). A utilização de novas técnicas alternativas, como a aplicação de reguladores vegetais ou mesmo subdoses de herbicidas, pode possibilitar a redução dos custos de manutenção de gramados. Além disso, o corte das folhas dos gramados através do procedimento de poda pode reduzir sua tolerância ao estresse ambiental, e os principais efeitos são perda de água, desenvolvimento de doenças, diminuição no armazenamento de carboidratos, aumento da intensidade de brotação e diminuição do crescimento das raízes e rizomas. McCullough et al. (2007) mencionam como desvantagem o fato de o corte vertical das folhas dos gramados de campo de golfe ter sido indicado como um agente causal do declínio de áreas de "greens" em alguns cultivares de gramas bermudas (Cynodon dactylon), e que estas requerem três semanas ou mais para recuperação da estética após o corte.

Os reguladores vegetais estão ganhando grande importância nos programas de manejo de gramados, uma vez que suprimem o crescimento vegetativo das plantas sem prejudicar sua qualidade visual e sem promover fitointoxicação, descoloração ou afinamento das folhas (Richardson, 2002; Ferrell et al., 2003; McCarty et al., 2004; Costa et al., 2009). Embora originalmente introduzidos para reduzir a frequência do número de corte e florescimento (Johnson, 1994), os reguladores vegetais podem também promover maior tolerância dos gramados ao estresse hídrico (Marcum \& Jian, 1997; Jlang \& Fry, 1998; Ervin \& Koski, 2001a; Bian et al., 2009) e aos efeitos de sombreamento (Qian et al., 1998; Qian \& Engelke, 1999).

A aplicação de reguladores vegetais para o manejo do desenvolvimento em gramados no Brasil ainda é considerada insignificante, seja em áreas esportivas, residenciais, industriais, de lazer ou em margens de rodovias. Uma prova disso é a baixíssima quantidade de trabalhos publicados por pesquisadores brasileiros 
e a falta de interesse da indústria química em manter ou financiar linhas de pesquisas direcionadas a esse mercado, considerado incipiente. Nesse sentido, a utilização de herbicidas seletivos e que promovam efeito supressivo sem prejudicar a qualidade visual dos gramados pode ser uma ferramenta de manejo interessante em razão do duplo propósito a ser atingido, diferentemente da singularidade dos reguladores de crescimento.

Este trabalho teve como objetivo avaliar o desenvolvimento das gramas Bermuda (C. dactylon), Esmeralda (Z. japonica) e São Carlos (Axonopus sp.) submetidas à aplicação de retardadores de crescimento e herbicidas em duas condições de luminosidade.

\section{MATERIAL E MÉTODOS}

O trabalho foi conduzido no período de setembro a dezembro de 2006, em estufas plásticas localizadas no campus urbano da Escola Superior de Agronomia de Paraguaçu Paulista - ESAPP, por meio de três experimentos, representados pelas gramas Bermuda (Cynodon dactylon), Esmeralda (Zoysia japonica) e São Carlos (Axonopus compressus).

O delineamento experimental utilizado foi o inteiramente casualizado, com 12 tratamentos e 4 repetições, dispostos em esquema fatorial $6 \times 2$ (seis condições de reguladores vegetal $\mathrm{x}$ duas condições de luminosidade) para cada espécie de grama. Os efeitos da aplicação dos reguladores de crescimento trinexapacethyl (250 $\mathrm{g} \mathrm{ha}^{-1}$ ), ethephon (300 $\mathrm{g} \mathrm{ha}^{-1}$ ) e dos herbicidas clethodim (12 $\left.\mathrm{g} \mathrm{ha}^{-1}\right)$, imazethapyr $\left(12,5 \mathrm{~g} \mathrm{ha}^{-1}\right)$, metsulfuron-methyl $\left(3,6 \mathrm{~g} \mathrm{ha}^{-1}\right) \mathrm{e}$ uma testemunha sem aplicação foram contrastados em duas condições de luminosidade, representadas pelo desenvolvimento das gramas em estufa plástica coberta com filme de polietileno transparente (luz total $=0 \%$ de sombreamento) e estufa plástica coberta com filme de polietileno transparente e tela de polipropileno (sombrite), com redução de 50\% da luminosidade (luz parcial $=50 \%$ de sombreamento). Para a condição sombreamento, as unidades experimentais foram acondicionadas em estufa plástica e dentro de armações de madeira com $1,5 \mathrm{~m}$ de altura do solo e 2,0 m de comprimento e largura, cobertas por tela de polipropileno (sombrite).
As unidades experimentais foram constituídas por vasos plásticos com capacidade de $10 \mathrm{~kg}$ de substrato, constituído pela mistura de dois terços de solo de textura arenosa com um terço de adubo orgânico curtido (esterco de curral). As mudas das gramas foram plantadas utilizando-se dois "plugs" por vaso, sendo estes mantidos em ambiente aberto antes da aplicação dos tratamentos, e efetuaram-se irrigações diárias.

Após as superficies das unidades experimentais serem cobertas pelas gramas, foi efetuado o corte uniforme das aparas das gramas a $5 \mathrm{~cm}$ de altura, em relação ao nivel do solo, dois dias antes da aplicação dos tratamentos. Para mensuração da altura do gramado, três hastes de madeira por vaso foram previamente inseridas no solo, para auxílio do nivelamento da altura de corte desejada e padronização das posteriores avaliações. Quanto às gramas Bermuda e São Carlos, também foram mantidos três estolões por vaso, para avaliação do crescimento em comprimento no sentido horizontal.

As aplicações dos tratamentos nos três experimentos foram feitas com solo úmido no dia 23/9/2006, dois dias após a operação de roçada para nivelamento da altura das gramas, utilizando-se pulverizador costal, pressurizado à pressão constante de $210 \mathrm{kPa}$ por $\mathrm{CO}_{2}$, com pontas XR 110.02, espaçadas de $0,5 \mathrm{~m}$, e consumo de calda de $200 \mathrm{~L} \mathrm{ha}^{-1}$. No momento das aplicações, as condições climáticas apresentavam em média temperatura de $26,9{ }^{\circ} \mathrm{C}$, umidade relativa do ar de $53 \%$ e velocidade do vento de $1,2 \mathrm{~km} \mathrm{~h}^{-1}$.

As características avaliadas para as três espécies de grama foram: fitointoxicação da parte aérea aos 7, 14, 21 e 28 DAA (dias após aplicação), por meio de notas visuais, em que 0\% corresponde à ausência de injúrias e 100\% à morte das plantas (SBCPD, 1995); crescimento médio em altura das gramas $(\mathrm{cm})$ aos $7,14,21,28,35,42,49,56$ e 63 DAA, obtido pela distância vertical entre a superficie roçada das folhas a $3 \mathrm{~cm}$ do solo e as novas pontas formadas em inclinação natural, utilizandose três amostragens aleatórias; massa seca da parte aérea e das raízes ( $\mathrm{g}$ por vaso) aos 63 DAA, assim como comprimento de estolões (cm), das gramas Bermuda e São Carlos; e número de inflorescências (no por vaso) apenas para a grama Bermuda aos 63 DAA. 
Os dados obtidos foram submetidos à análise de variância pelo teste $\mathrm{F}$, e as médias, comparadas pelo teste de Tukey a $5 \%$ de probabilidade. Os dados de altura da parte aérea das gramas foram submetidos à análise de regressão linear e polinomial quadrática. Os modelos adotados foram devido à explicação biológica, à significância do quadrado médio da regressão e aos coeficientes de regressão.

\section{RESULTADOS E DISCUSSÃO}

Os reguladores de crescimento e herbicidas promoveram maiores danos visuais na parte aérea das gramas mais pronunciados até $14 \mathrm{DAA}$; as injúrias não foram mais percebidas a partir de 28 DAA (Tabela 1). De modo geral, os sintomas de intoxicação caracterizam-se principalmente pela supressão do desenvolvimento vegetativo das gramas em diferentes intensidades, seguido por aspectos de amarelecimento ou clorose nas folhas, e/ou apenas pela redução da coloração verde em tonalidade e brilho, proporcionando coloração verde opaca aos limbos foliares. Nas gramas Bermuda (C. dactylon) e Esmeralda (Z. japonica), o trinexapac-ethyl promoveu necrosamento das bordas do limbo foliar, onde se efetuou o corte pela roçada, caracterizando um anel ou halo esbranquiçado nas pontas das folhas, não observado nas testemunhas.

Os produtos que mais prejudicaram as características visuais da grama Bermuda, na ausência de sombreamento, foram, em ordem decrescente: trinexapac-ethyl, imazethapyr e clethodim; para sombreamento em $50 \%$, destacaram-se como os mais fitotóxicos: metsulfuron-methyl, clethodim e trinexapacethyl (Tabela 1 e Figura 1). Os maiores niveis de danos visuais na grama Esmeralda foram observados quando se aplicou clethodim e imazethapyr na ausência de sombreamento, e metsulfuron-methyl e clethodim em sombreamento (Tabela 1 e Figura 1). Para o trinexapac-ethyl, os resutados corroboram os descritos por Qian et al. (1998), os quais constataram que aplicações sequenciais (480 $\mathrm{g} \mathrm{ha}^{-1}$ ) aumentaram a qualidade do gramado de Zoysia matrella, favorecendo a fotossintese em condição de sombreamento. Já para a grama São Carlos (A. compressus), o trinexapac-ethyl promoveu elevado nivel fitotóxico principalmente aos 7 DAA, diferindo significativamente dos demais tratamentos, que mantiveram médias inferiores a 13,9 e $2,7 \%$, respectivamente, para ausência e presença de sombreamento (Tabela 1 e Figura 1).

Com exceção do trinexapac-ethyl, a partir dos 14 DAA, os demais tratamentos mantiveram baixos niveis de intoxicação da grama São Carlos, quantificados respectivamente em média de 5,0 e 3,5\%, para ausência e presença de sombreamento, e próximo à ausência de danos aos 21 DAA.

Sintomas de fitointoxicação descritos como coloração púrpura e necroses de pontas e bordas das folhas da grama-batatais (P. notatum) foram registrados por Freitas et al. (1997) e Maciel et al. (2007), respectivamente, utilizando os herbicidas clethodim e sethoxydim; após 20 a 30 dias da aplicação, iniciou-se o processo de recuperação das injúrias dos gramados. De forma semelhante, Costa et al. (2010a,b) observaram que os herbicidas sethoxydim e metsulfuron-methyl promoveram intoxicação moderada a severa somente por um período de 7 a 26 DAA para as gramas Esmeralda, São Carlos e Gramabatatais, além da grama Santo Agostinho (Stenotaphrum secundatum). Nesse sentido, Christoffoleti \& Aranda (2002) mencionaram que o processo de seletividade de herbicidas em gramados depende da variedade de grama e do tipo de herbicida, não existindo uma regra única de recomendação, sendo, portanto, necessárias recomendações diferenciadas.

Todos os tratamentos promoveram redução significativa da altura das gramas até 63 DAA, independentemente da condição de luminosidade a que foram submetidos após aplicação (Figuras 2, 3 e 4 e Tabela 2). Apesar de o sombreamento ter favorecido o crescimento em altura das gramas, o trinexapac-ethyl apresentou o melhor desempenho na presença ou ausência de sombreamento; as reduções nas estaturas em relação às testemunhas aos 63 DAA foram, em média, de 65,1, 69,4 e $63,9 \%$, respectivamente, para as espécies Bermuda, Esmeralda e São Carlos. De acordo com Ervin et al. (2002), a aplicação de trinexapac-ethyl apresenta-se como prática de manejo efetiva da estatura de gramados de $Z$. japonica em áreas de baixa de luminosidade. 
Dessa forma, esses resultados corroboram os relatados por Costa et al. (2009) em trabalhos desenvolvidos em campo em condições brasileiras, que constataram a redução da necessidade de cortes de até 55 dias após a aplicação sequencial de trinexapac-ethyl $\left(56,5+56,5\right.$ e $113,0+113,0$ g ha $\left.^{-1}\right)$ em gramados de $A$. compressus, $S$. secundatum, $P$. notatume $Z$. japonica, assim como por Freitas et al. (2002) com $P$. notatum; em ambos os casos não foi constatado efeito deletério no aspecto visual das referidas espécies.

Tabela 1 - Fitointoxicação (\%) das plantas de gramas Bermuda (C. dactylon), Esmeralda (Z. japonica) e São Carlos (A. compressus) aos 7, 14 e 21 DAA dos tratamentos conduzidos em ausência de sombreamento (luz total) e 50\% de sombreamento (luz parcial)

\begin{tabular}{|c|c|c|c|c|c|c|}
\hline \multirow{2}{*}{ Tratamento } & \multicolumn{2}{|c|}{$7 \mathrm{DAA}$} & \multicolumn{2}{|c|}{14 DAA } & \multicolumn{2}{|c|}{$21 \mathrm{DAA}$} \\
\hline & Luz Total & Luz Parcial & Luz Total & Luz Parcial & Luz Total & Luz Parcial \\
\hline & \multicolumn{6}{|c|}{ C. dactylon } \\
\hline 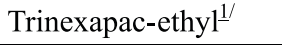 & $13,8 \mathrm{aA}$ & $0,0 \mathrm{cB}$ & $7,8 \mathrm{a}$ & $9,0 \mathrm{a}$ & $9,5 \mathrm{aA}$ & $8,0 \mathrm{aB}$ \\
\hline Ethephon $^{2 /}$ & $5,5 \mathrm{bcA}$ & $3,0 \mathrm{cA}$ & $3,3 \mathrm{~b}$ & $2,0 \mathrm{~cd}$ & $2,5 \mathrm{bA}$ & $3,0 \mathrm{cA}$ \\
\hline Clethodim $^{3 /}$ & $9,0 \mathrm{bB}$ & $12,5 \mathrm{bA}$ & $5,0 \mathrm{ab}$ & $5,0 \mathrm{bc}$ & $2,3 \mathrm{bB}$ & $4,5 \mathrm{bA}$ \\
\hline Imazethapyr $^{4 /}$ & $10,0 \mathrm{abA}$ & $8,3 \mathrm{bA}$ & $4,0 \mathrm{~b}$ & $5,8 \mathrm{~b}$ & $3,0 \mathrm{bA}$ & $0,0 \mathrm{~dB}$ \\
\hline Metsulfuron-methyl ${ }^{5 /}$ & $3,3 \mathrm{cdB}$ & $26,3 \mathrm{aA}$ & $3,0 \mathrm{bc}$ & $5,0 \mathrm{bc}$ & $0,0 \mathrm{cB}$ & $2,8 \mathrm{cA}$ \\
\hline Testemunha & $0,0 \mathrm{dA}$ & $0,0 \mathrm{cA}$ & $0,0 \mathrm{c}$ & $0,0 \mathrm{~d}$ & $0,0 \mathrm{cA}$ & $0,0 \mathrm{dA}$ \\
\hline $\mathrm{F}_{\mathrm{cal}}$ Produtos $(\mathrm{P})$ & \multicolumn{2}{|c|}{$44,06^{*}$} & \multicolumn{2}{|c|}{$29,44^{*}$} & \multicolumn{2}{|c|}{$176,76^{*}$} \\
\hline $\mathrm{F}_{\text {cal }}$ Ambiente $(\mathrm{A})$ & \multicolumn{2}{|c|}{$5,00^{*}$} & \multicolumn{2}{|c|}{$2,23^{\mathrm{NS}}$} & \multicolumn{2}{|c|}{$0,78^{*}$} \\
\hline $\mathrm{F}_{\text {cal }} \mathrm{P} \times \mathrm{A}$ & \multicolumn{2}{|c|}{$60,31^{*}$} & \multicolumn{2}{|c|}{$1,49^{\mathrm{NS}}$} & \multicolumn{2}{|c|}{$22,46^{*}$} \\
\hline \multirow[t]{2}{*}{$\mathrm{CV}(\%)$} & \multicolumn{2}{|c|}{28,81} & \multicolumn{2}{|c|}{34,97} & \multicolumn{2}{|c|}{22,08} \\
\hline & \multicolumn{6}{|c|}{ Z. japonica } \\
\hline 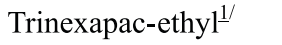 & $10,0 \mathrm{bA}$ & $0,0 \mathrm{~dB}$ & $5,5 \mathrm{bA}$ & $3,0 \mathrm{cB}$ & $3,0 \mathrm{aB}$ & $5,0 \mathrm{aA}$ \\
\hline Ethephon $^{2 /}$ & $13,8 \mathrm{bA}$ & $3,0 \mathrm{~dB}$ & $2,5 \mathrm{cA}$ & $2,5 \mathrm{cA}$ & $0,0 \mathrm{bB}$ & $2,5 \mathrm{bA}$ \\
\hline Clethodim $^{3 /}$ & $28,8 \mathrm{aA}$ & $12,5 \mathrm{bB}$ & $4,3 \mathrm{bcA}$ & $5,5 \mathrm{bA}$ & $3,0 \mathrm{aB}$ & $5,0 \mathrm{aA}$ \\
\hline Imazethapyr $^{4 /}$ & $27,5 \mathrm{aA}$ & $8,3 \mathrm{cB}$ & $3,0 \mathrm{cA}$ & $3,0 \mathrm{cA}$ & $2,5 \mathrm{aB}$ & $3,0 \mathrm{bA}$ \\
\hline Metsulfuron-methyl ${ }^{5 /}$ & $3,3 \mathrm{cB}$ & $26,3 \mathrm{aA}$ & $12,8 \mathrm{aA}$ & $20,8 \mathrm{aA}$ & $3,0 \mathrm{aB}$ & $5,0 \mathrm{aA}$ \\
\hline Testemunha & $0,0 \mathrm{cA}$ & $0,0 \mathrm{dA}$ & $0,0 \mathrm{dA}$ & $0,0 \mathrm{dA}$ & $0,0 \mathrm{bA}$ & $0,0 \mathrm{cA}$ \\
\hline $\mathrm{F}_{\mathrm{cal}}$ Produtos $(\mathrm{P})$ & \multicolumn{2}{|c|}{$130,05^{*}$} & \multicolumn{2}{|c|}{$389,65 *$} & \multicolumn{2}{|c|}{$416,40 *$} \\
\hline $\mathrm{F}_{\text {cal }}$ Ambiente $(\mathrm{A})$ & \multicolumn{2}{|c|}{$94,26^{*}$} & \multicolumn{2}{|c|}{$21,31^{*}$} & \multicolumn{2}{|c|}{$486,00^{*}$} \\
\hline $\mathrm{F}_{\text {cal }} \mathrm{P} \times \mathrm{A}$ & \multicolumn{2}{|c|}{$122,25 *$} & \multicolumn{2}{|c|}{$36,04^{*}$} & \multicolumn{2}{|c|}{$36,00^{*}$} \\
\hline $\mathrm{CV}(\%)$ & & & & & & 84 \\
\hline & & & A.co & ressus & & \\
\hline Trinexapac-ethyl $1^{1 /}$ & $27,5 \mathrm{aB}$ & $38,8 \mathrm{aA}$ & $6,5 \mathrm{aA}$ & $3,5 \mathrm{aB}$ & $10,5 \mathrm{aA}$ & $7,3 \mathrm{aB}$ \\
\hline Ethephon $^{2 /}$ & $11,5 \mathrm{bA}$ & $2,3 \mathrm{bB}$ & $6,8 \mathrm{aA}$ & $2,3 \mathrm{abB}$ & $4,8 \mathrm{bcA}$ & $3,0 \mathrm{bcA}$ \\
\hline Clethodim $^{3 /}$ & $16,8 \mathrm{bA}$ & $3,4 \mathrm{bB}$ & $6,0 \mathrm{aA}$ & $3,0 \mathrm{abB}$ & $6,5 \mathrm{bA}$ & $2,3 \mathrm{bcB}$ \\
\hline Imazethapyr $^{4 / /}$ & $14,8 \mathrm{bA}$ & $2,3 \mathrm{bB}$ & $4,0 \mathrm{aA}$ & $4,5 \mathrm{aA}$ & $3,5 \mathrm{bcA}$ & $4,5 \mathrm{abA}$ \\
\hline Metsulfuron-methyl ${ }^{5 /}$ & $12,5 \mathrm{bA}$ & $3,0 \mathrm{bB}$ & $6,3 \mathrm{aA}$ & $4,5 \mathrm{aA}$ & $2,3 \mathrm{cdA}$ & $0,0 \mathrm{cB}$ \\
\hline Testemunha & $0,0 \mathrm{cA}$ & $0,0 \mathrm{bA}$ & $0,0 \mathrm{bA}$ & $0,0 \mathrm{bA}$ & $0,0 \mathrm{dA}$ & $0,0 \mathrm{cA}$ \\
\hline $\mathrm{F}_{\mathrm{cal}}$ Produtos $(\mathrm{P})$ & & $82 *$ & & $81 *$ & & $38^{*}$ \\
\hline $\mathrm{F}_{\text {cal }}$ Ambiente(A) & & $35^{*}$ & & $49 *$ & & $49^{*}$ \\
\hline $\mathrm{F}_{\text {cal }} \mathrm{P} \times \mathrm{A}$ & & $75^{*}$ & & $82 *$ & & $90^{*}$ \\
\hline CV $(\%)$ & & & & & & \\
\hline
\end{tabular}

Obs.: ${ }^{1 /}$ Moddus $^{\circledR}$; ${ }^{2 /}$ Ethrel $^{\circledR ;} ;{ }^{3 /}$ Select ${ }^{\circledR ;} ;{ }^{4 /}$ Pivot $^{\circledR ;} ;{ }^{5 /}$ Ally $^{\circledR}$. Todos os produtos foram acrescidos de espalhante adesivo Assist ${ }^{\circledR}(0,5 \%$ v/v) na calda de pulverização. - DAA = dias após aplicação. Médias seguidas de mesma letra, minúscula na coluna e maiúscula na linha, não diferem estatisticamente entre si pelo teste de Tukey a $5 \%$. ${ }^{*}$ significativo $\mathrm{P}<0,05$; NS não significativo. 


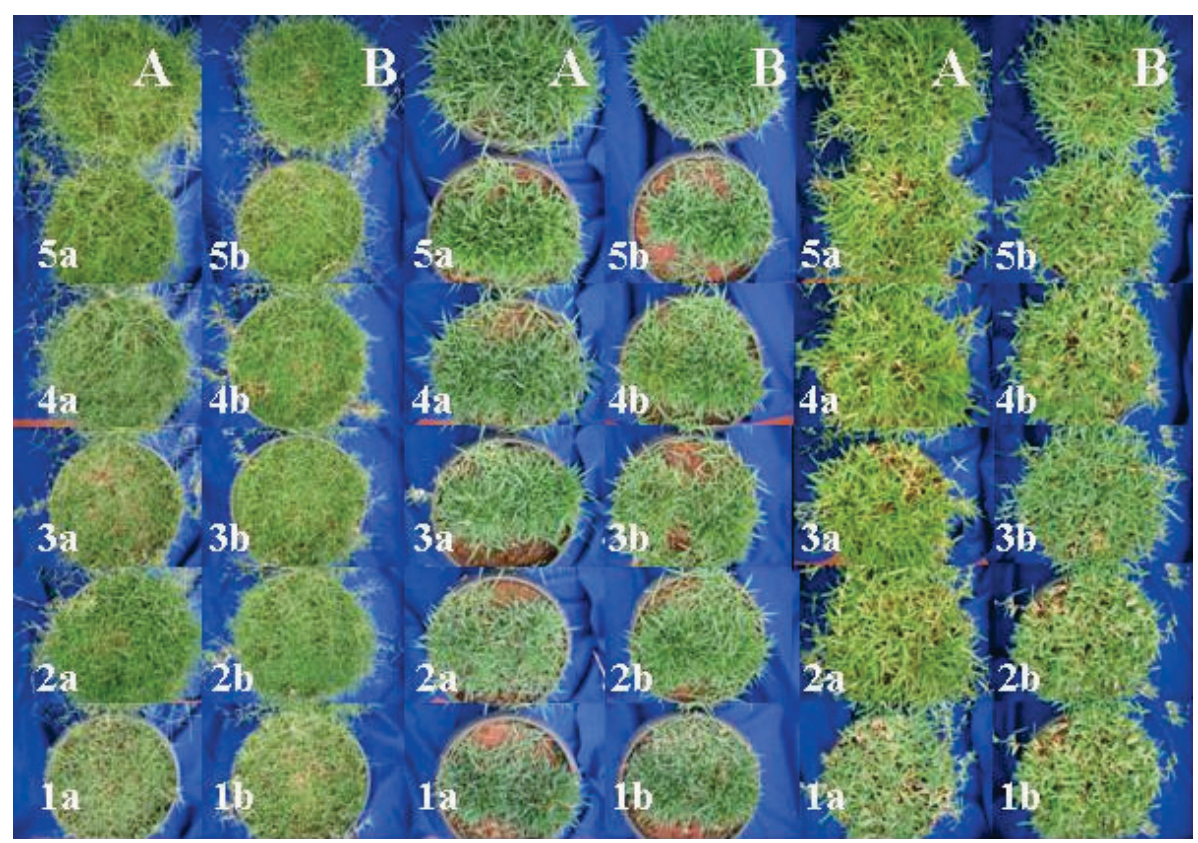

C. dactylon

Z. japonica

A. compressus

Figura 1 - Aspectos visuais das gramas Bermuda (C. dactylon), Esmeralda (Z. japonica) e São Carlos (A. compressus) aos 21 DAA, submetidas à aplicação dos reguladores de crescimento (1) trinexapacethyl (250 $\left.\mathrm{g} \mathrm{ha}^{-1}\right)$ e (2) ethephon (300 $\left.\mathrm{g} \mathrm{ha}^{-1}\right)$ e dos herbicidas (3) clethodim (12 $\left.\mathrm{g} \mathrm{ha}^{-1}\right)$, (4) imazethapyr $\left(12,5 \mathrm{~g} \mathrm{ha}^{-1}\right)$ e (5) metsulfuron-methyl (3,6 $\left.\mathrm{g} \mathrm{ha}^{-1}\right)$, conduzidos em ausência sombreamento (A) e $50 \%$ de sombreamento (B).

Segundo Johnson (1994), o corte em gramados de $C$. dactylon deve ser efetuado quando estes atingirem um terço de sua altura, ou seja, 33,3\% acima da altura de corte $(3 \mathrm{~cm})$. Desse modo, o trinexapac-ethyl se destacou pela elevada eficiência na supressão do crescimento das três gramas estudadas, reduzindo a necessidade de cortes por período superior a nove semanas, independentemente da espécie e condição de luminosidade a que foram submetidas após aplicação (Figuras 2, 3 e 4). Bush et al. (1998) também relataram que o trinexapac-ethyl, nas doses de 320 e $480 \mathrm{~g} \mathrm{ha}^{-1}$, reduziu o crescimento vegetativo e a emissão de inflorescência da grama Axonopus affins, evitando cortes por três e seis semanas, respectivamente. Gardner $\&$ Wherley (2005) constataram que o trinexapac-ethyl reduziu o número de cortes em torno de 35-50, 58-76 e $55-80 \%$, respectivamente, para as gramas Festuca ovina, Festuca arundinacea e Poa trivialis, quando submetidas à condição de baixa luminosidade, também corroborando os resultados obtidos no presente trabalho. Entretanto, é importante ressaltar que, principalmente para as gramas Bermuda e São Carlos, apesar de o trinexapac-ethyl ter sido o mais eficiente na redução de crescimento, também proporcionou o maior nivel de intoxicação inicial da parte aérea, com sintomas visuais persistindo até 21 DAA (Tabela 1 e Figura 1), corroborando assim os resultados de descoloração descritos para a grama Bermuda por Johnson (1997), Wiekco (1997) e Wiekco \& Couillard (1997).

De forma semelhante, os herbicidas clethodim e imazethapyr também reduziram a necessidade de cortes das gramas Bermuda e Esmeralda por periodo entre oito e nove semanas (Figuras 2 e 3), sobretudo em condições não sombreadas, confirmando os resultados de Freitas et al. (1997) e Maciel et al. (2007), com a vantagem de terem sido visualmente menos fitotóxicos, a partir dos 14 DAA. Para a grama São Carlos, além do trinexapacethyl, destacaram-se apenas para condição não sombreada o ethephon e metsulfuronmethyl, reduzindo a necessidade de cortes por periodo de nove semanas (Figura 4). 


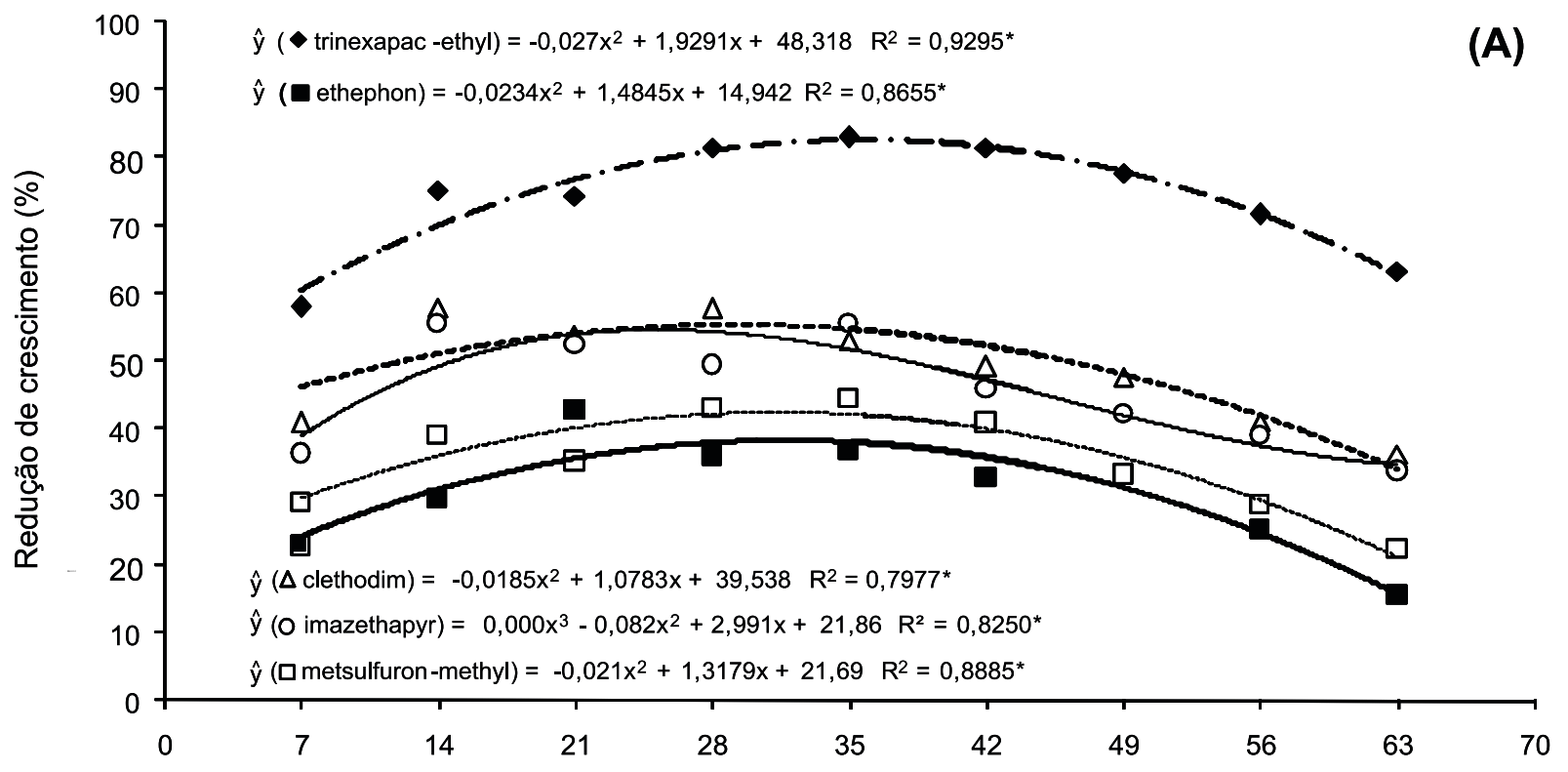

Dias após aplicação

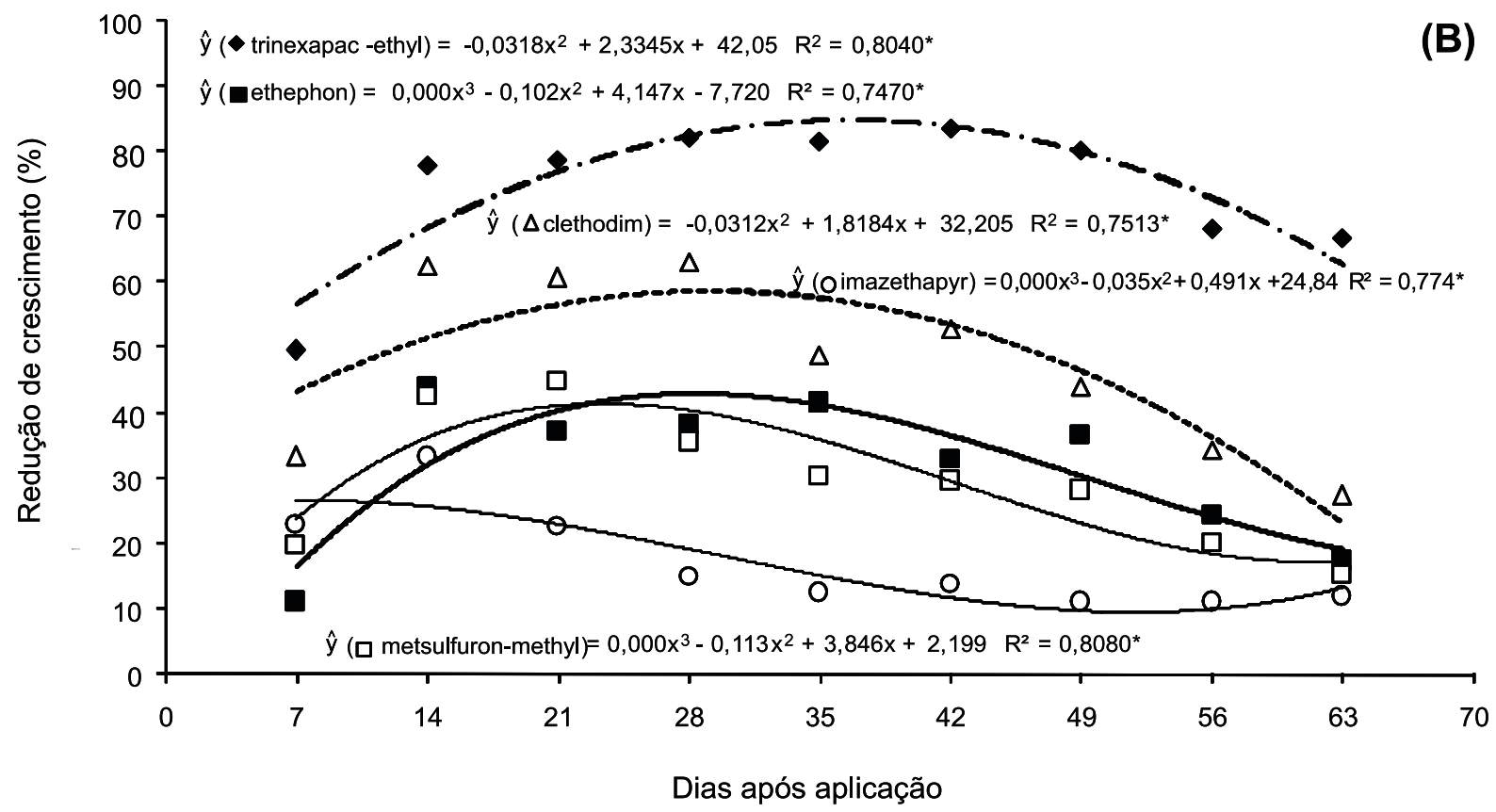

Figura 2 - Redução de crescimento (\%) da grama Bermuda (C. dactylon), em função da aplicação de reguladores de crescimento e subdoses de herbicidas, conduzida em ausência de sombreamento (A) e $50 \%$ de sombreamento $(\mathrm{B})$.

Rogers et al. (1987) também constataram baixa capacidade de supressão do crescimento da grama Bermuda com metsulfuron-methyl, confirmando com os resultados obtidos por Costa et al. (2010a,b) para as espécies $A$. compressus, $S$. secundatum e $P$. notatum.
Quanto ao desenvolvimento de estolões das gramas Bermuda e São Carlos, pode-se constatar que o crescimento em comprimento foi menos intenso quando se aplicou o trinexapacethyl (36,6 e 40,8\%) em relação aos demais tratamentos, nas condições de presença e 
ausência de sombreamento, respectivamente (Tabela 2). Os herbicidas clethodim, imazethapyr e metsulfuron-methyl também promoveram reduções do comprimento dos estolões em torno de 30,9, 18,3 e 25,7\%, respectivamente, para a grama Bermuda, e de 35,2, 34,5 e 4,6\% para a São Carlos, mas inferiores ao trinexapac-ethyl, independentemente da condição de luminosidade a que as gramas foram submetidas. De forma contrária, o ethephon não interferiu significativamente no desenvolvimento dos estolões das gramas Bermuda e São Carlos, assim como o metsulfuron-methyl para a grama
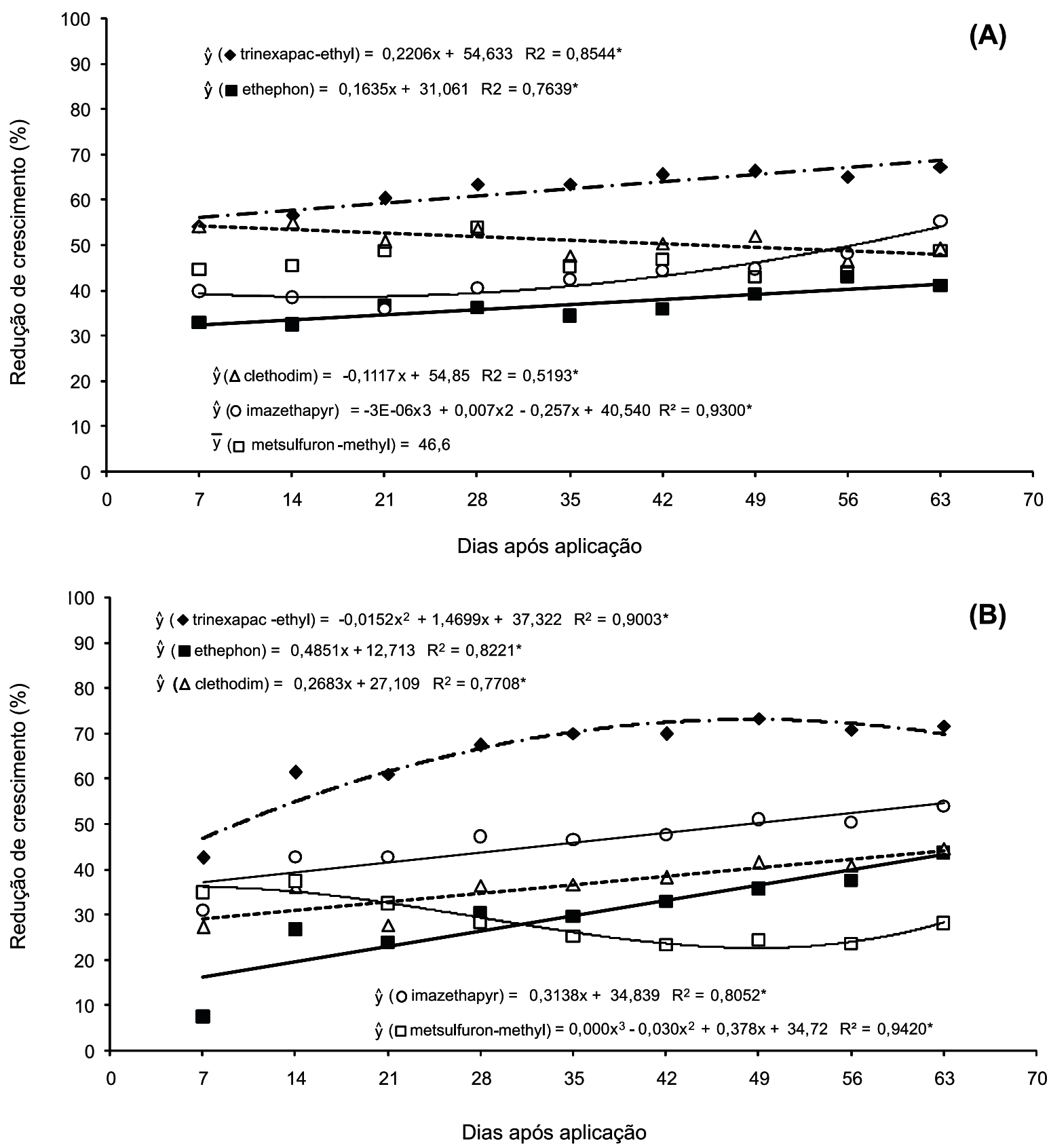

Figura 3 - Redução de crescimento (\%) da grama Esmeralda ( $Z$. japonica), em função da aplicação de reguladores de crescimento e subdoses de herbicidas, conduzida em ausência de sombreamento (A) e $50 \%$ de sombreamento (B). 
São Carlos. Todos os tratamentos apresentaram elevada capacidade de supressão do florescimento da grama Bermuda, porém apenas o trinexapac-ethyl, independentemente do sombreamento, e clethodim na ausência de sombreamento mostraram eficiência plena (100\%) na supressão de inflorescências até os 63 DAA (Tabela 2 e Figura 5).
Houve grande variação no comportamento fisiológico da massa seca da parte aérea e das raízes em relação aos tratamentos e espécies de gramas estudados, sendo principalmente influenciado pela condição de luminosidade a que elas foram submetidas (Tabela 3). Com exceção do imazethapyr, e de ethephon apenas na ausência de sombreamento, foram

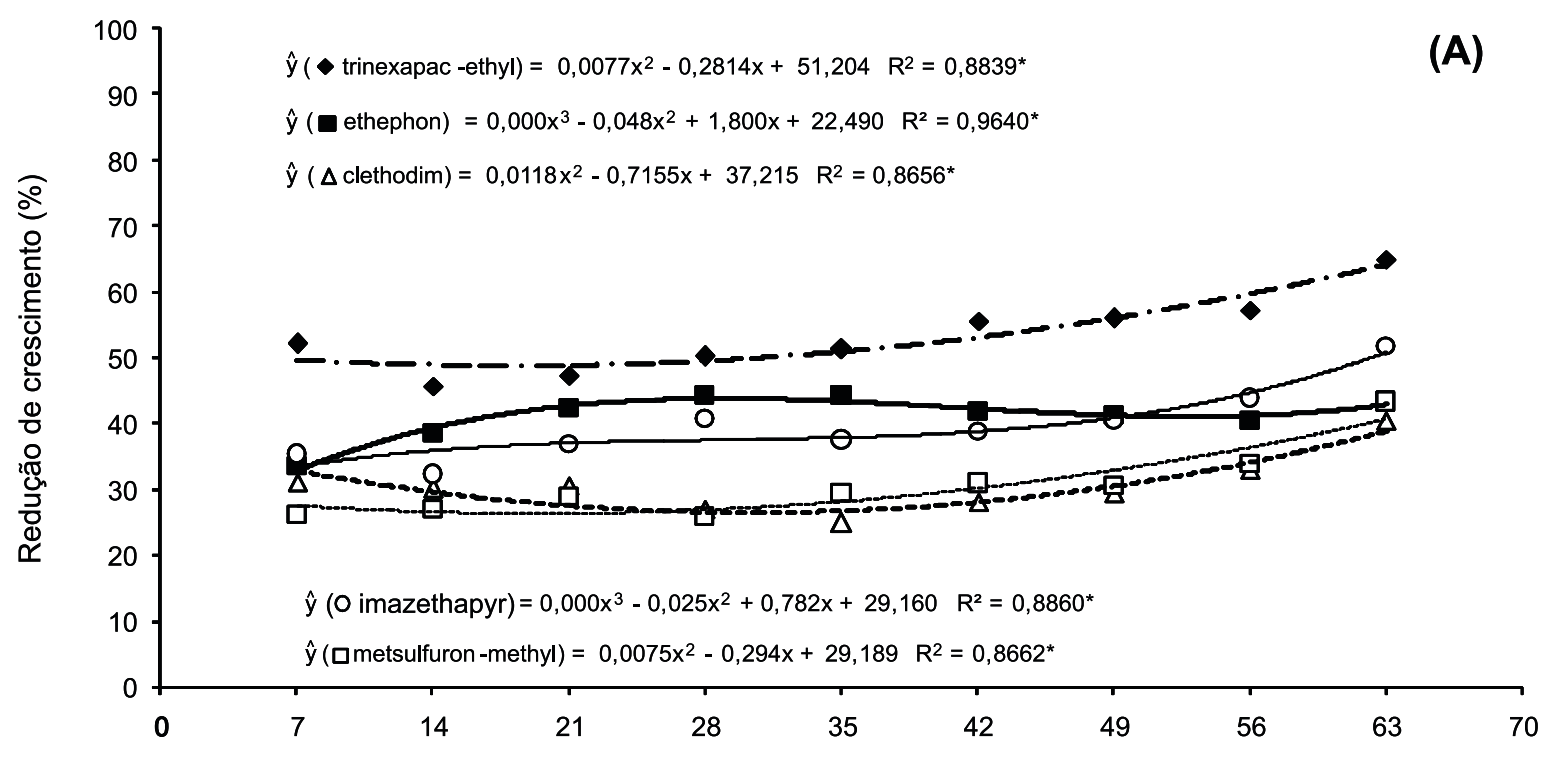

Dias após aplicação

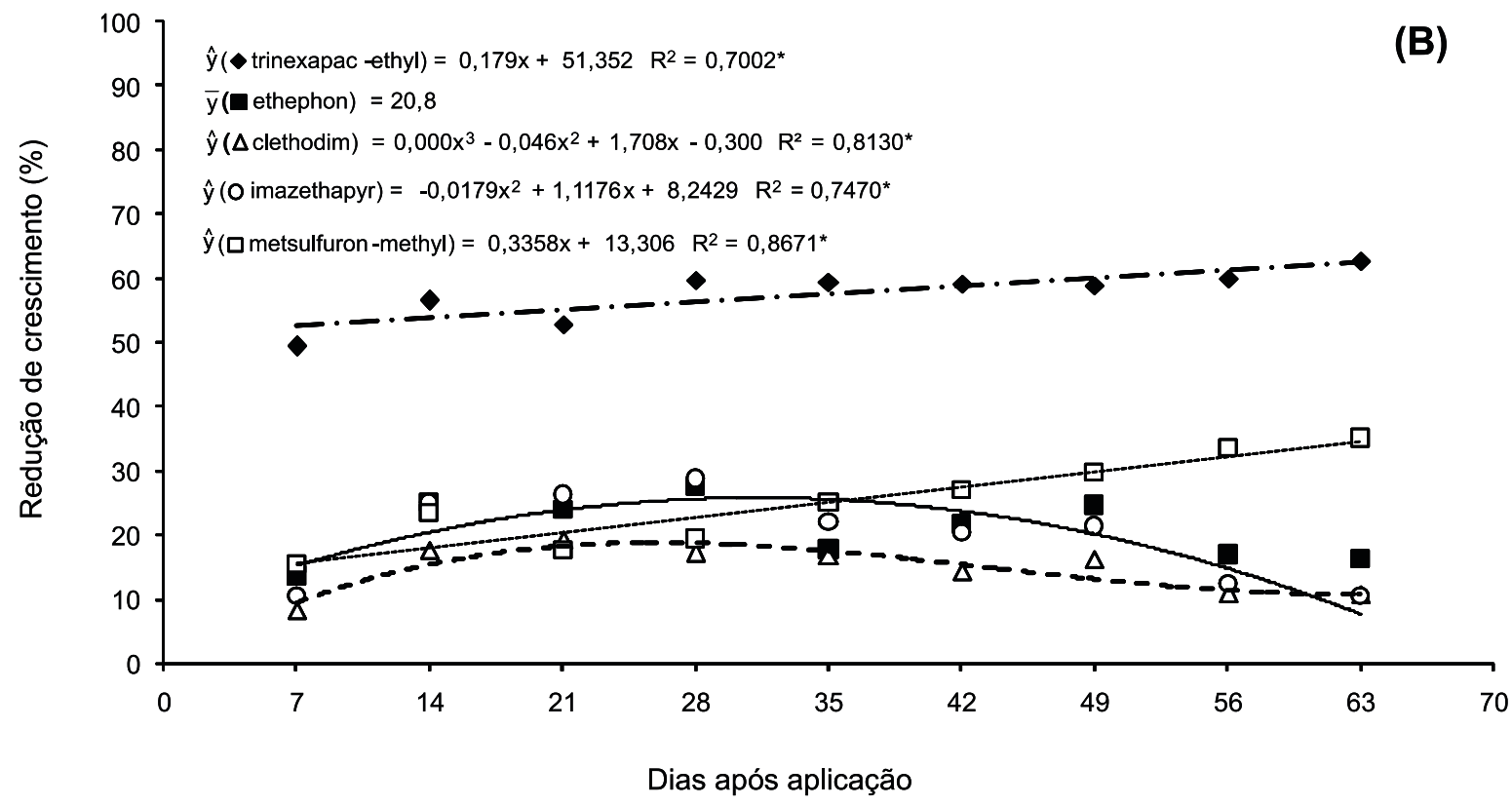

Figura 4 - Redução de crescimento (\%) da grama São Carlos (A. compressus), em função da aplicação de reguladores de crescimento e subdoses de herbicidas, conduzida em ausência de sombreamento (A) e $50 \%$ de sombreamento (B). 
Tabela 2 - Crescimento em altura $(\mathrm{cm})$ e comprimento $(\mathrm{cm})$ de estolões das plantas de gramas Bermuda (C. dactylon), Esmeralda (Z. japonica) e São Carlos (A. compressus) e número de inflorescências por vaso da grama Bermuda aos 63 DAA dos tratamentos conduzidos em ausência de sombreamento (luz total) e $50 \%$ de sombreamento (luz parcial)

\begin{tabular}{|c|c|c|c|c|c|c|}
\hline \multirow{3}{*}{ Tratamento } & \multicolumn{6}{|c|}{ Crescimento em altura de plantas $(\mathrm{cm})$} \\
\hline & \multicolumn{2}{|c|}{ C. dactylon } & \multicolumn{2}{|c|}{ Z. japonica } & \multicolumn{2}{|c|}{ A. compressus } \\
\hline & Luz Total & Luz Parcial & Luz Total & Luz Parcial & Luz Total & Luz Parcial \\
\hline Trinexapac-ethyl $1^{1 /}$ & $2,9 \mathrm{~dB}$ & $5,9 \mathrm{eA}$ & $4,7 \mathrm{dA}$ & $5,1 \mathrm{eA}$ & $5,0 \mathrm{dA}$ & $5,1 \mathrm{eA}$ \\
\hline Ethephon ${ }^{2 /}$ & $6,7 \mathrm{bB}$ & $14,7 \mathrm{cA}$ & $8,4 \mathrm{bB}$ & $10,1 \mathrm{cA}$ & $8,1 \mathrm{bB}$ & $11,5 \mathrm{cA}$ \\
\hline Clethodim ${ }^{3 /}$ & $5,1 \mathrm{cB}$ & $13,0 \mathrm{dA}$ & $7,3 \mathrm{bcB}$ & $9,9 \mathrm{cA}$ & $8,5 \mathrm{bB}$ & $12,3 \mathrm{bA}$ \\
\hline Imazethapyr $^{4 /}$ & $5,2 \mathrm{cB}$ & $15,7 \mathrm{bA}$ & $6,4 \mathrm{cB}$ & $8,2 \mathrm{dA}$ & $6,9 \mathrm{cB}$ & $12,3 \mathrm{bA}$ \\
\hline 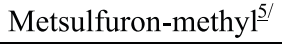 & $6,2 \mathrm{bB}$ & $15,1 \mathrm{bcA}$ & $7,3 \mathrm{~b} \mathrm{cB}$ & $12,9 \mathrm{bA}$ & $8,1 \mathrm{bB}$ & $8,9 \mathrm{dA}$ \\
\hline Testemunha & $7,9 \mathrm{aB}$ & $17,8 \mathrm{aA}$ & $14,3 \mathrm{aA}$ & $14,7 \mathrm{aA}$ & $9,5 \mathrm{aB}$ & $13,7 \mathrm{aA}$ \\
\hline $\mathrm{F}_{\text {cal }}$ Produtos $(\mathrm{P})$ & \multicolumn{2}{|c|}{$536,00^{*}$} & \multicolumn{2}{|c|}{$204,00^{*}$} & \multicolumn{2}{|c|}{$909,98^{*}$} \\
\hline $\mathrm{F}_{\text {cal }}$ Ambiente(A) & \multicolumn{2}{|c|}{$6.396,73^{*}$} & \multicolumn{2}{|c|}{$127,19^{*}$} & \multicolumn{2}{|c|}{$2.364,03 *$} \\
\hline $\mathrm{F}_{\text {cal }} \mathrm{P} \times \mathrm{A}$ & \multicolumn{2}{|c|}{$116,88^{*}$} & \multicolumn{2}{|c|}{$17,83^{*}$} & \multicolumn{2}{|c|}{$195,90^{*}$} \\
\hline CV $(\%)$ & \multicolumn{2}{|c|}{3,61} & \multicolumn{2}{|c|}{6,98} & \multicolumn{2}{|c|}{2,30} \\
\hline Tratamento & \multicolumn{2}{|c|}{$\begin{array}{l}\text { Comprimento de estolões } \\
\text { (C. dactylon })\end{array}$} & \multicolumn{2}{|c|}{$\begin{array}{l}\text { Comprimento de estolões } \\
\text { (A. compressus })\end{array}$} & \multicolumn{2}{|c|}{$\begin{array}{c}\mathrm{N}^{\mathrm{o}} \text { de inflorescências } \\
(\text { C. dactylon })\end{array}$} \\
\hline 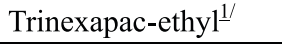 & $31,5 \mathrm{cA}$ & $23,7 \mathrm{~dB}$ & $32,2 \mathrm{~b}$ & $31,4 \mathrm{c}$ & $0,0 \mathrm{~d}$ & $0,0 \mathrm{c}$ \\
\hline Ethephon $^{2 /}$ & $49,1 \mathrm{aA}$ & $36,7 \mathrm{abB}$ & $53,1 \mathrm{a}$ & $55,4 \mathrm{a}$ & $4,3 \mathrm{c}$ & $2,0 \mathrm{c}$ \\
\hline Clethodim ${ }^{3 /}$ & $34,9 \mathrm{bcA}$ & $25,3 \mathrm{bcB}$ & $33,8 \mathrm{~b}$ & $35,8 \mathrm{bc}$ & $1,3 \mathrm{~cd}$ & $0,0 \mathrm{c}$ \\
\hline Imazethapyr $^{4 /}$ & $38,3 \mathrm{bA}$ & 32,9 abcA & $38,6 \mathrm{~b}$ & $31,8 \mathrm{c}$ & $1,8 \mathrm{~cd}$ & $1,5 \mathrm{c}$ \\
\hline Metsulfuron-methyl $^{5 /}$ & $41,1 \mathrm{bA}$ & $23,6 \mathrm{cdB}$ & $54,7 \mathrm{a}$ & $47,8 \mathrm{ab}$ & $8,3 \mathrm{~b}$ & $8,5 \mathrm{~b}$ \\
\hline Testemunha & $49,9 \mathrm{aA}$ & $37,2 \mathrm{abB}$ & $58,4 \mathrm{a}$ & $49,0 \mathrm{a}$ & $16,8 \mathrm{a}$ & $15,0 \mathrm{a}$ \\
\hline $\mathrm{F}_{\text {cal }}$ Produtos $(\mathrm{P})$ & \multicolumn{2}{|c|}{$28,68^{*}$} & \multicolumn{2}{|c|}{$26,64^{*}$} & \multicolumn{2}{|c|}{$127,41^{*}$} \\
\hline $\mathrm{F}_{\text {cal }}$ Ambiente $(\mathrm{A})$ & \multicolumn{2}{|c|}{$78,38^{*}$} & \multicolumn{2}{|c|}{$3,80^{\mathrm{NS}}$} & \multicolumn{2}{|c|}{$3,88^{\mathrm{NS}}$} \\
\hline $\mathrm{F}_{\text {cal }} \mathrm{P} \times \mathrm{A}$ & \multicolumn{2}{|c|}{$2,75^{*}$} & \multicolumn{2}{|c|}{$1,54^{\mathrm{NS}}$} & \multicolumn{2}{|c|}{$0,88^{\mathrm{NS}}$} \\
\hline CV $(\%)$ & \multicolumn{2}{|c|}{10,19} & \multicolumn{2}{|c|}{13,35} & \multicolumn{2}{|c|}{31,17} \\
\hline
\end{tabular}

Obs.: ${ }^{1 /}$ Moddus $^{\circledR} ;{ }^{2 /}$ Ethrel $^{\circledR} ;{ }^{3 /}$ Select ${ }^{\circledR} ;{ }^{4 /}$ Pivot $^{\circledR} ;{ }^{\circledR} /$ Ally $^{\circledR}$. Todos os produtos foram acrescidos do espalhante adesivo Assist ${ }^{\circledR}$ na calda de pulverização. - DAA = dias após aplicação. Médias seguidas de mesma letra, minúscula na coluna e maiúscula na linha, não diferem estatisticamente entre si pelo teste de Tukey a $5 \%$. * significativo $\mathrm{P}<0,05$; Ns não significativo.

verificadas reduções médias da massa seca da parte aérea da grama Bermuda aos 63 DAA, na ordem de $30,1,27,2$ e $27,3 \%$, respectivamente, para trinexapac-ethyl, clethodim e metsulfuron-methyl. Para a grama Esmeralda, de forma semelhante à grama Bermuda, apenas o ethephon na ausência de sombreamento não reduziu a massa seca da parte aérea aos 63 DAA, ao contrário dos demais tratamentos, em que a redução do crescimento dessa espécie foi, em média, de 37,8, 31,9, 41,0 e $38,7 \%$, respectivamente, para trinexapacethyl, clethodim, imazethapyr e metsulfuronmethyl. Quanto à grama São Carlos, não foram constatadas diferenças significativas entre produtos e testemunhas, havendo apenas desenvolvimento inferior da massa seca da parte aérea na presença de sombreamento com trinexapac-ethyl e ethephon. Para as gramas Bermuda e Esmeralda, a condição de sombreamento proporcionou incremento e redução média de massa seca da parte aérea de 28,7 e $21,2 \%$, respectivamente.

Para produção de massa seca das raízes na ausência de sombreamento, apenas o metsulfuron-methyl não apresentou redução do desenvolvimento da grama Bermuda, ao contrário do trinexapac-ethyl, ethephon, clethodim e imazethapyr, que causaram, 


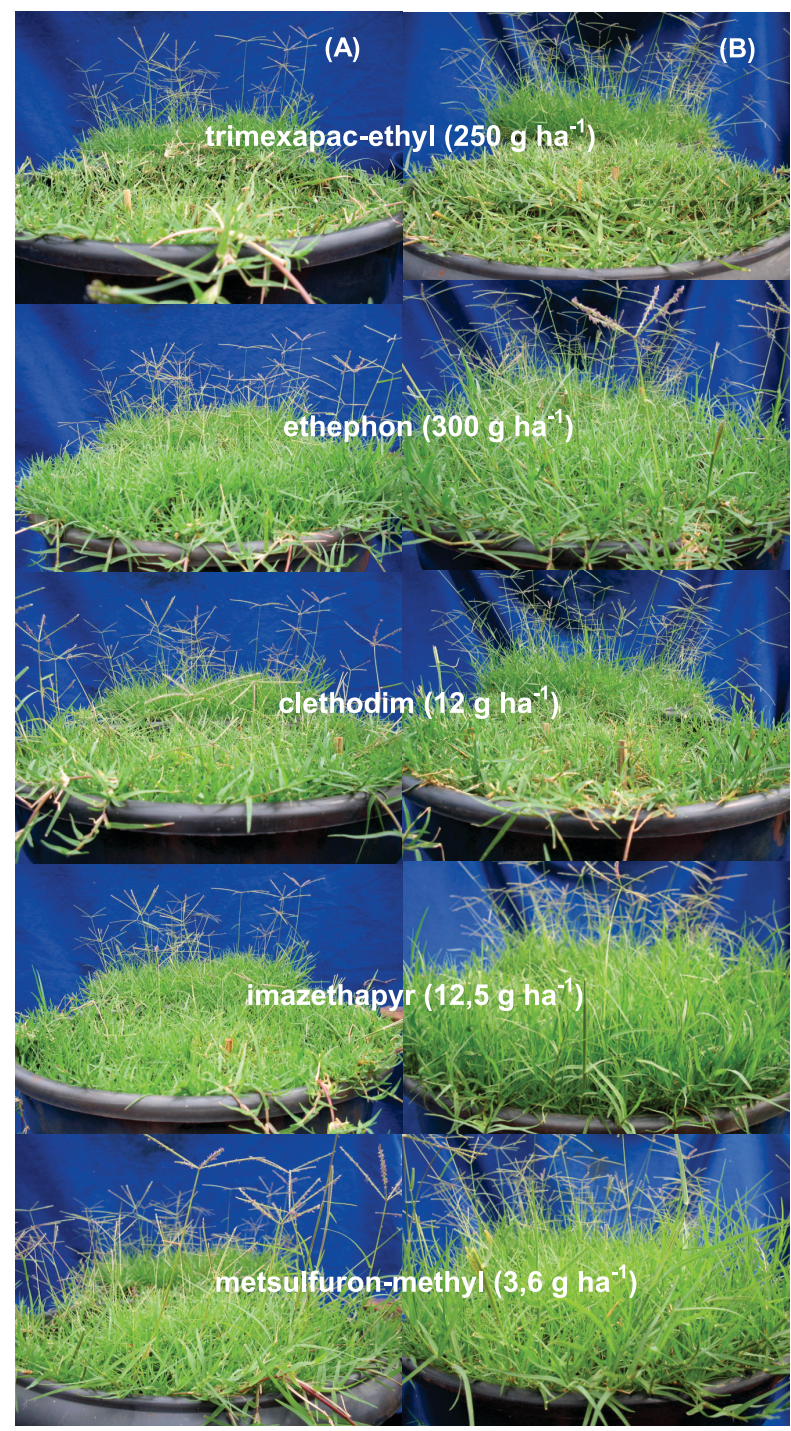

Figura 5 - Aspectos visuais do desenvolvimento vegetativo e reprodutivo da grama Bermuda (C. dactylon) aos 63 DAA, caracterizando os vasos frontais submetidos à aplicação de trinexapac-ethyl, ethephon, clethodim, imazethapyr e metsulfuron-methyl, e das testemunhas ao fundo com inflorescência, conduzidos em ausência de sombreamento (A) e $50 \%$ de sombreamento (B).

respectivamente, reduções médias de 15,3, $22,9,33,1$ e 18,7\% para grama Bermuda; de 23,3, 27,4, 25,4 e 43,3\% para grama Esmeralda; e de 19,3, 46,7, 19,2 e 28,4\% para grama São Carlos (Tabela 3). Para as gramas Esmeralda e São Carlos, a redução de massa seca das raízes na ausência de sombreamento com metsulfuron-methyl foi da ordem de 22,6 e $49,0 \%$, respectivamente. Na condição sombreada, as reduções da massa seca das raízes não foram significativas apenas para clethodim e imazethapyr nas gramas Bermuda e São Carlos, assim como para clethodim e metsulfuron-methyl em Esmeralda, o que caracteriza para esses herbicidas um diferencial seletivo positivo sobre as referidas espécies. Trinexapac-ethyl reduziu significativamente a produção de massa seca das raízes das três gramas estudadas, na ausência $(19,4 \%)$ e presença de sombreamento $(24,8 \%)$, com exceção da grama São Carlos em condição de sombreamento. Estudos sobre os efeitos de trinexapac-ethyl no desenvolvimento de raizes de gramados têm gerado resultados conflitantes. Beasley et al. (2005) relataram que a aplicação de trinexapac-ethyl reduziu em torno de $30 \%$ o comprimento e a área de superficie de raízes na base dos perfilhos da grama Poa pratensis. Do mesmo modo, Goss et al. (2002) observaram, em condição de baixa luminosidade, que o trinexapac-ethyl reduziu a produção de raízes por perfilho da grama Agrostis stolonifera cv. Penncross por período de sete semanas, corroborando os resultados do presente trabalho. De forma contrária, Ervin \& Koski (2001b) e Fagerness \& Yelverton (2001) não encontraram influência do trinexapac-ethyl na produção de raízes das gramas Poa pratensis e Agrostis stolonfera, assim como McCarty et al. (2004) constataram aumento de $45 \%$ para $C$. dactylon, diferentemente dos reguladores de crescimento paclobutrazol e flurprimidol, que reduziram a produção de raízes.

Tendo em vista o exposto, conclui-se que os melhores resultados de redução do desenvolvimento vegetativo das espécies de grama Bermuda (C. dactylon), Esmeralda (Z. japonica) e São Carlos (A. compressus) e emissão da inflorescência da grama Bermuda (C. dactylon), na ausência ou presença de sombreamento, foram obtidos com trinexapac-ethyl, clethodim e imazethapyr. Entretanto, em situações em que a preservação da estética do gramado é fundamental, os herbicidas clethodim (12 $\left.\mathrm{g} \mathrm{ha}^{-1}\right)$ e imazethapyr $\left(12,5 \mathrm{~g} \mathrm{ha}^{-1}\right)$ podem substituir o trinexapac-ethyl, devido à maior seletividade e eficiência na supressão do florescimento para gramados da espécie Bermuda (C. dactylon). Os tratamentos 
Tabela 3 - Massa seca da parte aérea e de raízes (g por vaso) das gramas Bermuda (C. dactylon), Esmeralda (Z. japonica) e São Carlos (A. compressus) aos 63 DAA dos tratamentos em diferentes condições de luminosidade

\begin{tabular}{|c|c|c|c|c|c|c|}
\hline \multirow{3}{*}{ Tratamento } & \multicolumn{6}{|c|}{ Massa seca da parte aérea ( $\mathrm{g}$ por vaso) } \\
\hline & \multicolumn{2}{|c|}{ C. dactylon } & \multicolumn{2}{|c|}{ Z. japonica } & \multicolumn{2}{|c|}{ A. compressus } \\
\hline & Luz Total & Luz Parcial & Luz Total & Luz Parcial & Luz Total & Luz Parcial \\
\hline 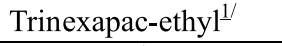 & $35,2 \mathrm{bB}$ & $45,9 \mathrm{bA}$ & $60,6 \mathrm{bA}$ & $30,5 \mathrm{cdB}$ & $69,2 \mathrm{~A}$ & $55,2 \mathrm{~B}$ \\
\hline Ethephon $^{2 /}$ & $44,3 \mathrm{abA}$ & $47,7 \mathrm{bA}$ & $78,5 \mathrm{aA}$ & $40,2 \mathrm{bcdB}$ & $65,7 \mathrm{~A}$ & $50,9 \mathrm{~B}$ \\
\hline Clethodim ${ }^{3 /}$ & $38,4 \mathrm{bA}$ & $46,1 \mathrm{bA}$ & $54,3 \mathrm{bcA}$ & $45,4 \mathrm{bB}$ & $65,1 \mathrm{~A}$ & $58,7 \mathrm{~A}$ \\
\hline Imazethapyr $^{4 /}$ & $42,4 \mathrm{abB}$ & $58,3 \mathrm{aA}$ & $56,8 \mathrm{bcA}$ & $29,6 \mathrm{~dB}$ & $58,6 \mathrm{~A}$ & $55,9 \mathrm{~A}$ \\
\hline${\text { Metsulfuron-methyl }{ }^{5 /}}$ & $37,4 \mathrm{bB}$ & $46,9 \mathrm{bA}$ & $48,5 \mathrm{cA}$ & $41,2 \mathrm{bcB}$ & $58,5 \mathrm{~A}$ & $58,4 \mathrm{~A}$ \\
\hline Testemunha & $52,3 \mathrm{aB}$ & $63,7 \mathrm{aA}$ & $81,9 \mathrm{aA}$ & $64,5 \mathrm{aB}$ & $60,3 \mathrm{~A}$ & $64,4 \mathrm{~A}$ \\
\hline $\mathrm{F}_{\text {cal }}$ Produtos $(\mathrm{P})$ & \multicolumn{2}{|c|}{$9,83 *$} & \multicolumn{2}{|c|}{$44,24 *$} & \multicolumn{2}{|c|}{$2,27^{\mathrm{NS}}$} \\
\hline $\mathrm{F}_{\text {cal }}$ Ambiente $(\mathrm{A})$ & \multicolumn{2}{|c|}{$32,05^{*}$} & \multicolumn{2}{|c|}{$227,25^{*}$} & \multicolumn{2}{|c|}{$19,74^{*}$} \\
\hline $\mathrm{F}_{\text {cal }} \mathrm{P} \times \mathrm{A}$ & \multicolumn{2}{|c|}{$0,96 *$} & \multicolumn{2}{|c|}{$12,64 *$} & \multicolumn{2}{|c|}{$5,99 *$} \\
\hline $\mathrm{CV}(\%)$ & \multicolumn{2}{|c|}{12,83} & \multicolumn{2}{|c|}{9,38} & \multicolumn{2}{|c|}{7,3} \\
\hline \multirow{2}{*}{ Tratamento } & \multicolumn{6}{|c|}{ Massa seca de raízes (g por vaso) } \\
\hline & \multicolumn{2}{|c|}{ C. dactylon } & \multicolumn{2}{|c|}{ Z. japonica } & \multicolumn{2}{|c|}{ A. compressus } \\
\hline Trinexapac-ethy $1^{1 /}$ & $56,6 \mathrm{bA}$ & $42,8 \mathrm{bB}$ & $59,1 \mathrm{bA}$ & $43,5 \mathrm{bB}$ & $53,9 \mathrm{bA}$ & $37,0 \mathrm{bcB}$ \\
\hline Ethephon ${ }^{2 /}$ & $51,5 \mathrm{bcA}$ & $43,8 \mathrm{bB}$ & $56,0 \mathrm{bA}$ & $31,3 \mathrm{cB}$ & $35,6 \mathrm{cA}$ & $33,7 \mathrm{cA}$ \\
\hline Clethodim ${ }^{3 /}$ & $44,7 \mathrm{cA}$ & $45,3 \mathrm{abA}$ & $57,5 \mathrm{bA}$ & $60,7 \mathrm{aA}$ & $54,0 \mathrm{bA}$ & $45,3 \mathrm{abB}$ \\
\hline Imazethapyr $^{4 /}$ & $54,3 \mathrm{bA}$ & $50,8 \mathrm{abA}$ & $43,7 \mathrm{bA}$ & $37,8 \mathrm{bcA}$ & $47,8 \mathrm{bA}$ & $46,3 \mathrm{aA}$ \\
\hline Metsulfuron-methyl $^{5 /}$ & $68,5 \mathrm{aA}$ & $34,4 \mathrm{cB}$ & $59,7 \mathrm{bA}$ & $63,0 \mathrm{aB}$ & $34,1 \mathrm{cA}$ & $37,9 \mathrm{bcA}$ \\
\hline testemunha & $66,8 \mathrm{aA}$ & $53,3 \mathrm{aB}$ & 77, 1 aA & $62,0 \mathrm{aB}$ & $66,8 \mathrm{aA}$ & $42,2 \mathrm{abcB}$ \\
\hline $\mathrm{F}_{\text {cal }}$ Produtos $(\mathrm{P})$ & \multicolumn{2}{|c|}{$14,91^{*}$} & \multicolumn{2}{|c|}{$54,02 *$} & \multicolumn{2}{|c|}{$26,36^{*}$} \\
\hline $\mathrm{F}_{\text {cal }}$ Ambiente(A) & \multicolumn{2}{|c|}{$120,70 *$} & \multicolumn{2}{|c|}{$53,91 *$} & \multicolumn{2}{|c|}{$45,53 *$} \\
\hline $\mathrm{F}_{\text {cal }} \mathrm{P} \times \mathrm{A}$ & \multicolumn{2}{|c|}{$20,78^{*}$} & \multicolumn{2}{|c|}{$13,57^{*}$} & \multicolumn{2}{|c|}{$12,92 *$} \\
\hline CV $(\%)$ & \multicolumn{2}{|c|}{7,41} & \multicolumn{2}{|c|}{7,91} & & \\
\hline
\end{tabular}

Obs.: ${ }^{1 /}$ Moddus $^{\circledR} ;{ }^{2 /}$ Ethrel $^{\circledR}$; $3 /$ Select ${ }^{\circledR} ;{ }^{4 /}$ Pivot $^{\circledR} ;{ }^{5 /}$ Ally ${ }^{\circledR}$. Todos os produtos foram acrescidos do espalhante adesivo Assist ${ }^{\circledR}$ na calda de pulverização. - DAA = dias após aplicação. Médias seguidas de mesma letra, minúscula na coluna e maiúscula na linha, não diferem estatisticamente entre si pelo teste de Tukey a $5 \%$. ${ }^{*}$ significativo $\mathrm{P}<0,05$; Ns não significativo.

ethephon (300 $\left.\mathrm{g} \mathrm{ha}^{-1}\right)$ e metsulfuron-methyl $\left(3,6 \mathrm{~g} \mathrm{ha}^{-1}\right)$ destacaram-se na redução do desenvolvimento vegetativo $(15,5$ e $26,7 \%)$ e da matéria seca das raízes $(34,7$ e $33,9 \%)$ da grama São Carlos (A. compressus), principalmente na condição de sombreamento, sendo caracterizados como as melhores opções para a espécie, quando comparado ao trinexapacethyl.

Além disso, ressalta-se a necessidade de novos estudos com reguladores de crescimento e subdoses de herbicidas para outras espécies de gramas cultivadas, com o objetivo de ampliar os conhecimentos entre a interação da seletividade e manutenção dos gramados, e que viabilizem recomendações eficientes e seguras, com embasamento em informações científicas geradas em condições brasileiras.

\section{LITERATURA CITADA}

BIAN, X.; MEREWITZ, E.; HUANG, B. Effects of trinexapac-ethyl on drought responses in creeping bentgrass associated with water use and osmotic adjustment. J. Am. Soc. Hortic. Sci., v. 134, p. 505-510, 2009.

BEASLEY, J. S.; BRANHAM, B. E.; ORTIZ-RIBBING, L. M. Trinexapac-ethyl affects Kentucky Bluegrass root architecture. Hort Sci., v. 40, n. 6, p. 1539-1542, 2005

BUSH, E. W. et al. Controlling growth of common carpetgrass using selected plant growth regulators. Hort Sci., v. 33, n. 4, p. 704-706, 1998. 
COSTA, N. V. et al. Características morfológicas de gramas em resposta a ação de trinexapac-ethyl. Planta Daninha, v. 27, n. 1, p. $113-122,2009$

COSTA, N. V. et al. Seletividade de herbicidas aplicados nas gramas Santo Agostinho e Esmeralda. Planta Daninha, v. 28 , n. 1, p. 139-148, 2010a.

COSTA, N. V. et al. Seletividade de herbicidas aplicados na grama Batatais e na grama São Carlos. Planta Daninha, v. 28, n. 2 , p. $365-374,2010 b$.

ERVIN, E. H.; KOSKI, A. J. Trinexapac-ethyl effects on Kentucky Bluegrass evapotranspiration. Crop Sci., v. 41, n. 4, p. 247-250, 2001a.

ERVIN, E. H.; KOSKI, A. J. Kentucky bluegrass growth responses to trinexapac-ethyl, traffic, and nitrogen.

Crop Sci., v. 41, p. 1871-1877, 2001 b.

ERVIN, E. H. et al. Trinexapac-ethyl restricts shoot growth and prolongs stand density of 'Meyer' zoysiagrass fairway under shade. Hort Sci., v. 37, n. 3, p. 502-505. 2002.

FAGEMESS, M. J.;YELVERTON, F. H. Plant growth regulator and mowing height effects on seasonal root growth of penncross creeping bentgrass. Crop Sci., v. 41, p. $1901-1905,2001$

FREITAS, F. C. L. et al. Efeitos do trinexapac-ethyl sobre o crescimento e florescimento da grama-batatais.

Planta Daninha, v. 20, n. 3, p. 477-486, 2002.

FERRELL, J. A. et al. Seashore paspalum response to trinexapac-ethyl and paclobutrazol. Hort Sci., v. 38, n. 4, p. 605-606, 2003.

FREITAS, F. C. L. et al. Influência de cimetacarb e clethodim como reguladores de crescimento em gramado. In: CONGRESSO BRASILEIRO DA CIÊNCIA DAS PLANTAS DANINHAS, 21., 1997, Caxambu: Resumos... Caxambu: SBCP, 1997. p. 325.

GARDNER, D. S.; WHERLEY, B. G. Growth response of three turfgrass species to nitrogen and trinexapac-ethyl in shade. Hort Sci., v. 40, n. 6, p. 1911-1915, 2005.

MACIEL, C. D. G. et al. Comportamento do gramado submetido aos herbicidas clethodim e sethoxydim. R. Bras. Hortic. Ornam., v. 13, n. 2, p. 161-168, 2007.

McCARTY, L. B. et al. St. Augustinegrass response to plant growth retardants. Crop Sci., v. 44, p. 1323-1329, 2004.
McCUllough, P. E. et al. Trinexapac-ethyl application regimens influence growth, quality, and performance of Bermuda Grass and Creeping Bentgrass putting greens. Crop Sci., v. 47, n. 5, p. 247-250, 2007.

GOSS, R. M. et al. Trinexapac-ethyl and nitrogen effects on creeping bentgrass grown under reduced light conditions.

Crop Sci., v. 42, p. 472-479, 2002.

QIAN, Y. L. et al. Trinexapac-ethyl restricts shoot growth and improves quality of "Diamond" Zoysiagrass under shade Hort Sci., v. 33, n. 6, p. 1019-1022, 1998.

QIAN, Y. L.; ENGELKE, M. C. Influence of trinexapac-ethey on "Diamond" Zoysiagrass in a shade environment. Crop Sci., v. 39, p. 202-208, 1999.

JIANG, H.; FRY, J. Drought responses of perennial ryegrass treat plant growth regulators. Hort Sci., v. 33, n. 2, p. 270-273, 1998.

JONHSON, B. J. influence of plant-growth regulators and mowing on to bahiagrasses. Agron. J., v. 86, n. 5, p. 805-810, 1994.

JONHSON, B. J. Growth of 'Tifway' bermudagrass following application of nitrogen and iron with trinexapacethyl. Hort Sci., v. 32, n. 2, p. 241-242, 1997.

MARCUM, K. B.; JANG, H. Effects of plant growth regulators on tall fescue rooting and water use. J. Turfgrass Manag., v. 2, n. 2, p. 13-27, 1997.

ROGERS III, J. N.; MILLER, E. M.; KING, J. W. Growth retardation of bermudagrass with metsulfuron methyl and sulfometuron methyl. Agron. J., v. 79, p. 225-229, 1987.

RICHARDSON, M. D. Turf quality and freezing tolerance of Tifway bermudagrass as affected by late season nitrogen and trinexapac-ethyll. Crop Sci., v. 42, p. 1612-1626, 2002.

SOCIEDADE BRASILEIRA DA CIÊNCIA DAS PLANTAS DANINHAS - SBCPD. Procedimentos para instalação, avaliação e análise de experimentos com herbicidas. Londrina: $1995.42 \mathrm{p}$.

WIECKO, G. Response of 'Tifway' bermudagrass to trinexapac-ethyl. J. Turfgrass. Manag., v. 2, p. 29-36, 1997.

WIECKO, G.; COUILLARD, A. Response of 'Tifway' bermudagrass to trinexapac-ethyl and chelated iron. J. Turfgrass. Manag., v. 2, p. 15-21, 1997. 